\title{
The breast of parous women without cancer has a different genomic profile compared to those with cancer
}

\author{
GABRIELA A. BALOGH ${ }^{1}$, JOSE RUSSO ${ }^{1}$, DANIEL A. MAILO ${ }^{1}$, REBECCA HEULINGS ${ }^{1}$, \\ PATRICIA A. RUSSO ${ }^{1}$, PETER MORRISON ${ }^{2}$, FATHIMA SHERIFF $^{1}$, IRMA H. RUSSO ${ }^{1}$ \\ and THE FCCC HOSPITAL NETWORK PARTICIPANTS ${ }^{3}$ \\ ${ }^{1}$ Breast Cancer Research Laboratory, Fox Chase Cancer Center, Philadelphia, PA; \\ ${ }^{2}$ Department of Biostatistics, Biodiscovery, Inc., San Diego, CA, USA
}

Received May 24, 2007; Accepted July 6, 2007

\begin{abstract}
Our studies are aimed at determining whether pregnancy induces a specific genomic signature in the postmenopausal breast that is responsible for the protective effect elicited by this physiological process. For this purpose we designed a study to compare the gene expression profiles in normal breast tissue from parous postmenopausal women with (case) and without (control) breast cancer. We have used breast samples from 18 parous controls and 41 parous cases. The epithelium and the interlobular stroma were dissected using laser capture microdissection and the RNA of each compartment and each sample was isolated, amplified using PCR methodology, and hybridized to cDNA glass-microarrays containing 40,000 genes, placing the human reference RNA in the green channel ( $\mathrm{Cy} 3)$ and the breast tissue samples in the red channel (Cy5). The normalization and statistical analysis of the expression data were carried out by using the LIMMA software package for the R programming environment which provides functions to summarize the results using the linear model perform hypothesis tests and adjust the p-values for multiple testing. We were able to identify 126 genes that were upregulated and 103 that were downregulated in the parous control group. There were only 56 genes differentially expressed in the interlobular stroma in the parous control
\end{abstract}

Correspondence to: Dr Jose Russo, Breast Cancer Research Laboratory, Fox Chase Cancer Center, 333 Cottman Ave., Philadelphia, PA 19111, USA

E-mail: j_russo@fccc.edu

Network participants: ${ }^{3}$ Emily Penman and Nicholas J. Petrelli, Helen F. Graham Cancer Center, Christiana Care Health System; Angela Lanfranchi, Somerset Medical Center, 110 Red Hill Avenue, Somerville, NJ 08876; Kathryn Evers, Diagnostic Imaging and Monica Morrow, Department of Surgical Oncology, FCCC, Philadelphia, PA, USA

Key words: parous postmenopausal women, laser capture microdissection, breast cancer group in relation to the other group of women under study. The gene categories that were overrepresented in the breast epithelium of the parous control breast are related to apoptosis, DNA repair, response to exogenous agents and transcription regulation. In the present study we demonstrate that full-term pregnancy imprints a specific genomic signature in the breast epithelium of postmenopausal parous control women that is significantly different from women who have developed cancer. This genomic signature induced by pregnancy could help to predict in which women parity is protective.

\section{Introduction}

Breast cancer is the most common neoplastic disease in women and accounts for up to one third of all new cancer cases in North American women (1). There is substantial evidence that breast cancer risk relates to endocrinologic and reproductive factors. Breast cancer development is strongly dependent on the ovary and on endocrine conditions modulated by ovarian function, such as early menarche, late menopause, and parity (1-4). Epidemiological findings indicate that a lifetime risk decrease has been observed in parous women whose first pregnancy was completed before age 24 (2-6). However, the protection conferred by early first full-term pregnancy does not occur in all women. We have postulated that a cluster of genes associated with early first full-term pregnancy would be absent or modified in the breast of high-risk women, i.e., nulliparous women with cancer and also in parous women who developed cancer (7-9). Furthermore, those genes whose expression may be affected by pregnancy and that can be proven to be functionally relevant in protecting the breast from cancer development could serve as markers for evaluating cancer risk in large populations. In the present study we demonstrate that early first full-term pregnancy induces a genomic signature that is specific for the parous breast and that is different from that in those who are parous and have cancer.

\section{Materials and methods}

Sample collection. In this study we have included postmenopausal women who underwent breast biopsies (excluding fine needle biopsies) at FCCC or the participant's hospital 
Christiana Care and Somerset Medical Center between October 1, 2002 and December 30, 2006. Each participant had signed their respective informed consent forms that were approved by each Institution's Human Subjects Protection Committee. Women were 50+ years old and postmenopausal, defined as at least one year since last menses if menopause occurred naturally. We have excluded from our study women in which both ovaries were removed or with a history of cancer other than non-melanoma skin cancer, women taking medications that could interfere with the study protocol such as estrogens (including Tamoxifen and Raloxifene), progestins, androgens, prednisone, thyroid hormones, or insulin, and women with Alzheimer's disease or severe cognitive deficit who were unable to give informed consent.

Breast tissue specimens. A total of 59 histologically normal breast tissues from 18 parous controls and 41 parous cases were analyzed and microdissected for the epithelium from type 1 lobules $(10,11)$ and the interlobular stroma by using the laser capture microdissection (LCM) system (12). All the tissues were collected and fixed in $70 \%$ ethanol within 10 min of surgical removal. The tissues were embedded in paraffin. Eight-micron sections were obtained and stained with hematoxylin and eosin, followed by 5 -min dehydration steps in 70,95 and 100\% ethanol. Once air-dried the sections were laser microdissected with an AutoPix 1000 LCM system from Arcturus Engineering (Mountain View, CA). We captured epithelial cells from the type 1 lobules and interlobular stroma from each breast sample.

RNA isolation, labeling and cDNA human microarrays. The RNA was isolated by placing the LCM cap in $50 \mu 1$ of Trizol reagent and following procedures previously described (12). The probes were constructed using direct labeling of the random hexamer primer and following protocols described elsewhere (12). The probes were cleaned with a QIA-quick PCR purification kit (Qiagen); PB buffer (500 $\mu 1$ from kit) was added into the Cy3- and Cy5-labeled products. The samples were applied to QIA-quick columns, which were centrifuged at 13,000 rpm $(16,000 \mathrm{x} \mathrm{g})$ for $1 \mathrm{~min}$, after which the flow-through was discarded. The column was washed by adding $750 \mu \mathrm{l}$ of $80 \%$ cold ethanol, and spun again for $1 \mathrm{~min}$, and the flow-through was discarded. The washing was repeated twice, and the columns were spun again to remove residual ethanol. The collected material was diluted in $30 \mu 1$ of DEPCwater heated at $70^{\circ} \mathrm{C}$ followed by 3 -min incubation at $70^{\circ} \mathrm{C}$. Columns were then centrifuged at 13,000 rpm $(16,000 \mathrm{x} \mathrm{g})$ for $1 \mathrm{~min}$, and the elution step was performed only once. The eluted material was partially dried in a vacuum centrifuge and the volume was adjusted to $15 \mu 1$ of hybridization buffer [containing 20X saline-sodium citrate (SSC) and $0.6 \mu 1$ of $10 \%$ (wt/vol) SDS], then the probes were denatured at $95^{\circ} \mathrm{C}$ for $3 \mathrm{~min}$ and centrifuged for $3 \mathrm{~min}$ at 13,000 rpm. The products were pipetted onto arrays, coverslipped, and the slides were placed in a hybridization chamber (Gene Machine). Arrays were incubated in a water bath at $42^{\circ} \mathrm{C}$ for $16-18 \mathrm{~h}$, and subsequently washed with $0.5 \mathrm{X} \mathrm{SSC}, 0.01 \%$ (wt/vol) SDS, followed by $0.06 \mathrm{X} \mathrm{SSC}$, at room temperature for $10 \mathrm{~min}$ each. The slides were spun for $8 \mathrm{~min}$ at $800 \mathrm{rpm}(130 \mathrm{x} \mathrm{g})$ at room temperature.
Array scanning. Arrays were read with an Affymetrix 428 fluorescent scanner (MWG, CA) at $10-\mu \mathrm{m}$ resolution and variable photomultiplier tube (PMT) voltage settings to obtain the maximal signal intensities with $<1 \%$ (wt/vol) probe saturation. The resulting images were analyzed using ImaGene software version 4.2 (Biodiscovery, CA). The glass microarrays were hybridized in all the cases placing the amplified RNA from the breast samples in the red channel (labeled with Cy5) and the amplified RNA from human universal reference (Stratagene, Inc, CA) in the green channel (labeled with Cy3). We studied gene expression in the 59 patients by triplicate using cDNA microarrays, which were prepared by robotically spotting 40,000 human cDNA on mirror glass slides (NCIsupported Microarray Facility from Fox Chase Cancer Center). The cDNA included approximately 28,000 different genes that represented characterized human proteins and 10,000 identified by ESTs, the rest were controls and blank spots. The identities of the cDNA had been sequence-verified. Each hybridization compared Cy5-labelled cDNA reverse transcribed from amplified RNA isolated from each patient with the Cy3labelled cDNA reverse transcribed from a universal human reference amplified RNA sample. The reference sample was used in all the hybridizations employing the same probe for all of them in order to have an equal and common reference for the experiment. The fluorescent probes were performed in triplicate and after checking the quality, replicates from the same sample were combined and re-distributed into 3 separate tubes in order to have an identical replicate. Equal amounts of fluorescent probes were used to hybridize the cDNA microarrays.

Data analysis. Normalization and statistical analysis of the expression data were carried out by using the LIMMA software package for the $\mathrm{R}$ programming environment $(13,14)$. This package contains a number of analysis methods not found in other software. Local background subtraction usually produces log-ratios that are very variable at low intensities. Because it was desired to detect differential expression for genes that might not necessarily be highly expressed, filtering out lowintensity spots was avoided. Instead, a strategy of background correction was used that avoids exaggerated variability of log-ratios for low-intensity spots. Background correction was performed by using the 'normexp' method in LIMMA to adjust the local median background estimates. This strategy is similar to the background correction method used by the popular RMA software for Affymetrix data (15). It avoids problems with background estimates that are greater than foreground values and ensures that there were no missing or negative corrected intensities. An offset of 100 was used for both channels to further damp down the variability of logratios for low-intensity spots. The resulting log-ratios were normalized by using the print-tip group Lowess method with a span of 0.4, as recommended by Smyth and Speed (16). Here and elsewhere, the small number of spots that were manually marked as 'bad' on visual inspection of the scanned arrays were filtered out of the analysis, while spots that were flagged as 'not found' by GENESIGHT were kept in the analysis but downweighted. The arrays used in these experiments were from four different print runs that were all printed with the same elements but with slightly different print layouts. The 
arrays from the different print runs were therefore normalized separately and the normalized expression data were combined and aligned by probe for subsequent analysis. After normalization of the data, each microarray had similar distributions in order to eliminate the microarray effect once we detected the gene expression of certain genes.

The basic statistic used for significance analysis is the moderated t-statistic, which is computed for each probe and for each contrast. This has the same interpretation as an ordinary t-statistic except that the standard errors have been moderated across genes, i.e., shrunk towards a common value, using a simple Bayesian model. This has the effect of borrowing information from the ensemble of genes to aid with the same inference about each individual gene (17). Moderated t-statistics lead to p-values in the same way that ordinary t-statistics do except that the degrees of freedom are increased, reflecting the greater reliability associated with the smoothed standard errors. The most popular form of adjustment is 'fdr' which is Benjamini and Hochberg's method to control the false discovery rate (19). The meaning of fdr adjusted pvalues is as follows. If all genes with a p-value below a threshold, e.g. of 0.05 , are selected as differentially expressed, then the expected proportion of false discoveries in the selected group is controlled to be less than the threshold value, in this case $5 \%$. The B-statistic is the log-odds that the gene is differentially expressed (15). For example, if $\mathrm{B}=1.5$, the odds of differential expression $(1.5)=4.48$; i.e., $\sim 4.5: 1$. The probability that the gene is differentially expressed is 4.48 / $(1+4.48)=0.82$. A B-statistic of zero corresponds to a $50-50$ chance that the gene is differentially expressed. The B-statistic is automatically adjusted for multiple testing by assuming that $1 \%$ of the genes, or some other percentage specified by the user referring to empirical Bayes (17-19), is expected to be differentially expressed. The p-values and B-statistics will normally rank genes in the same order. In fact, if the data contains no missing values or quality weights, then the order will be precisely the same. As with all model-based methods, the p-values depend on normality and other mathematical assumptions which are never completely precise for microarray data. It has been argued that the p-values are useful for ranking genes even in the presence of large deviations from the assumptions. The B-statistic probabilities depend on the same assumptions but require in addition a prior estimate for the proportion of differentially expressed genes. The p-values may be preferred to the B-statistics because they do not require this prior knowledge (20).

\section{Results}

Identification of differentially expressed genes in breast epithelium. Genes whose expression changes were considered to be statistically significant using established algorithms, and whose expression changed by at least 1.2-fold as a result of being a case, were selected for further analysis (19). This combined analytic approach has previously been shown to be capable of identifying differentially expressed genes with high sensitivity and specificity (19). We were able to identify gene sequences differentially expressed (t-test with false discovery rate $\mathrm{p}<0.05)$ in epithelium from the parous breast control compared to that from the parous breast cancer cases. We
Table I. Upregulated genes in the parous control breast epithelia.

\begin{tabular}{ll}
\hline Apoptosis- GO:0006915; GO:0042985 & \\
BAX & 2.6500 \\
TIA1 & 1.5600 \\
TRAF1 & 1.7200 \\
TRADD & 1.4200 \\
CRADD & 1.8900 \\
PPM1F & 1.3500
\end{tabular}

$\begin{array}{ll}\text { Cell adhesion- GO:0007155; GO:0016337; GO:0007160 } & \\ \text { SEMA5A } & 1.810 \\ \text { ICAM3 } & 1.7000 \\ \text { EVA1 } & 1.2500 \\ \text { FBLN5 } & 1.7900 \\ \text { FNBP4 } & 1.2900 \\ \text { SDK1 } & 1.2600 \\ \text { NRP1 } & 1.2500\end{array}$

Signal transduction- GO:0007165; GO:0007242; GO:0016055; GO:0008277; GO:0007186

$\begin{array}{ll}\text { EMR2 } & 1.5100 \\ \text { ANK2 } & 1.2500 \\ \text { IRS1 } & 1.2900 \\ \text { CNIH2 } & 1.2500 \\ \text { CHN2 } & 1.3000 \\ \text { LRP5 } & 1.4000 \\ \text { GIT1 } & 1.3500 \\ \text { GALR2 } & 1.2000 \\ \text { Cell cycle and growth- GO:0000067; GO:0000074 } & \\ \text { DNAJA2 } & 1.5600 \\ \text { HIPK2 } & 1.8700 \\ \text { RBBP6 } & 1.5800 \\ \text { DCTN1 } & 1.4800\end{array}$

Response to exogenous agents- GO:0008152; GO:0006805; GO:0045454; GO:0042113; GO:0006725; GO:0006950; GO:0006979; GO:0006954; GO:0006952

$\begin{array}{ll}\text { RDH11 } & 1.6428 \\ \text { EPHX1 } & 1.7800 \\ \text { TXNRD1 } & 1.9200 \\ \text { IGBP1 } & 1.3800 \\ \text { CBARA1 } & 1.3800 \\ \text { TIRAP } & 1.3800 \\ \text { SCARA3 } & 1.3900 \\ \text { GSTT1 } & 1.2400 \\ \text { C10orf59 } & 1.9300 \\ \text { NAT2 } & 1.5000\end{array}$

Cell transport- GO:0006810; GO:0006811; GO:0006812; GO:0015031; GO:0005794; GO:003001; GO:0006826; GO:0006813; GO:0006406

TPR

TLOC1

1.6300

GALNT10

1.4700

ARMC1 
Table I. Continued.

\begin{tabular}{ll}
\hline TRPM1 & 1.2400 \\
CLCN6 & 1.3500 \\
HRB & 1.4000 \\
SHKBP1 & 1.6300 \\
TTYH1 & 1.6000 \\
SLC19A3 & 1.6400 \\
SLC22A9 & 1.5600 \\
Chromatin modification- GO:0016568; GO:0007001 & \\
HIST1H2AC & 1.2700 \\
SETD1A & 1.8700
\end{tabular}

Development-morphogenesis- GO:0007275; GO: 0030154; GO:0007399

$\begin{array}{ll}\text { DVL2 } & 2.0669 \\ \text { LTBP4 } & 1.9901 \\ \text { DOPEY2 } & 2.5697 \\ \text { EFNB3 } & 1.6402 \\ \text { DGCR14 } & 2.3211 \\ \text { FGF11 } & 2.2619\end{array}$

DNA repair- GO:0006284; GO:0006281; GO: 0000731; GO:0006298; GO:0006310

$\begin{array}{ll}\text { RAD51L3 } & 1.9200 \\ \text { ERCC8 } & 1.2500 \\ \text { POLD3 } & 1.5000 \\ \text { ANKRD17 } & 1.780 \\ \text { TSN } & 1.940 \\ \text { NTHL1 } & 1.9200 \\ \text { TREX1 } & 1.5400\end{array}$

Miscellaneous process- GO:0005887; GO:0016043; GO:0006936; GO:0005887; GO:00031012

$\begin{array}{ll}\text { CEACAM4 } & 1.6500 \\ \text { DIAPH3 } & 2.2219 \\ \text { SSPN } & 1.5900 \\ \text { THSD4 } & 1.8500\end{array}$

RNA processing- GO:0006396; GO:0006364; GO:0007600; GO:0008033; GO:0000245

$\begin{array}{ll}\text { DDX17 } & 3.024 \\ \text { EIF4A3 } & 1.710 \\ \text { TTC8 } & 1.8722 \\ \text { PUS7 } & 1.4400 \\ \text { POP7 } & 1.540 \\ \text { SIP1 } & 1.7400\end{array}$

Metabolism- GO:0030201; GO:0008152; GO:0007206; GO:0016042; GO:0006559; GO:0006796

HS3ST4

DBT

HOMER1

SIDT2

FAH

DHDDS

ACSS 1

PTPRB
Table I. Continued.

Protein biosynthesis and metabolism- GO:0006470; GO:0008104; GO:0006464; GO:0006468; GO:0006412; GO:0006457

$\begin{array}{ll}\text { PTPRC } & 1.3209 \\ \text { VPS13C } & 2.3952 \\ \text { LOX } & 3.6726 \\ \text { TTLL5 } & 3.2004 \\ \text { PTPN21 } & 1.2500 \\ \text { MGC42105 } & 1.2400 \\ \text { RPL9 } & 1.2600 \\ \text { GRPEL1 } & 1.2500 \\ \text { BACE2 } & 1.2400\end{array}$

Proteolysis and ubiquitination- GO:0006508; GO:0006511; GO:0006512; GO:0006397; GO:0006398; GO:0016567

$\begin{array}{ll}\text { CTSB } & 2.1571 \\ \text { DPP3 } & 1.5400 \\ \text { PEPD } & 1.420 \\ \text { EDD1 } & 1.6632 \\ \text { RNF44 } & 1.3200 \\ \text { ATE1 } & 1.2600 \\ \text { HNRPR } & 1.2500 \\ \text { GEMIN4 } & 1.3000\end{array}$

Transcription- GO:0000122; GO:0006355; GO:0006350; GO:0006357; GO:0006366

BPTF

2.000

SUPT5H

2.150

SOX10

1.9300

PCAF

1.2500

FOXK2

1.2550

KCNIP3

1.3000

PIAS1

1.3100

RFXAP

1.2400

ZNF16

1.2340

ID4

2.100

GTF2B

1.539

ZNF26

1.534

ZNF498

2.0074

ZNF544

1.2500

ZNF710

1.9023

BAZ2A

1.2450

HOXD1 1.2600

HIRA $\quad 1.2600$

TLE3

1.2500

ZNF268

1.5000

ZNF275

1.2800

Biological process unknown- GO:0000004

FBLN2

1.835

DHX57

2.9587

1.2400

$\begin{array}{ll}\text { WDR44 } & 1.8603 \\ \text { ENTPD3 } & 1.2600\end{array}$

ORMDL1 3.0238

ANKRD12 1.8890

TMEM27 1.7801

DKFZP434A0131 
found that 126 genes were upregulated (Table I) and 103 were downregulated (Table II) in the parous control group, with respect to the parous breast cancer group.

Gene functional category analysis. There are four major biological processes that were overrepresented in the parous control group; apoptosis, DNA repair, response to exogenous agents and transcription regulation. The apoptosis process in the parous control group contained six genes: BAX, BCL2associated X protein; TIA1, cytotoxic granule-associated RNA binding protein; TNF receptor-associated factor 1; TRADD, TNFRSF1A-associated via death domain; CRADD, CASP2 and RIPK1 domain containing adaptor with death domain; and PPM1F, protein phosphatase $1 F$ (PP2C domain containing) (Table I). However only two genes were downregulated in the parous control group; Transformed $3 T 3$ cell double minute 4-p53 binding protein (mouse) (Mdm4) and Programmed cell death 5 (PDCD5). There are also two antiapoptotic genes, Baculoviral IAP repeat-containing 6 (apollon) and BCL2-associated athanogene, that were downregulated in the parous control group (Table II). This indicates that the programmed cell death is a signature prevalently expressed in the parous control group.

The DNA repair process was also overrepresented in the parous control breast epithelia containing seven genes that were significantly upregulated (Table I): RAD51-like 3 (S. cerevisiae); Excision repair cross-complementing rodent repair deficiency, complementation group 8; Polymerase (DNA-directed), $\Delta 3$, accessory subunit; Ankyrin repeat domain 17; Translin; Nth endonuclease III-like 1 (E. coli); and Three prime repair exonuclease 1 .

The third biological process overrepresented in the parous control group was the cluster of genes related to cell response either in the immunosurveillance category or response to genotoxic agents (Table I), such as Retinol dehydrogenase 11 (all-trans/9-cis/11-cis) (RDH11); Epoxide hydrolase 1, microsomal (xenobiotic)( EPHX1); Thioredoxin reductase 1 (TXNRD1); Immunoglobulin (CD79A) binding protein 1 (IGBP1); Calcium binding atopy-related autoantigen 1 (CBARA1); Toll-interleukin 1 receptor (TIR) domain containing adaptor protein (TIRAP); Scavenger receptor class A, member 3 (SCARA3); Glutathione S-transferase $\theta 1$ (GSTT1); Chromosome 10 open reading frame 59 (C10orf59); and $\mathrm{N}$-acetyltransferase 2 (arylamine $\mathrm{N}$-acetyltransferase) (NAT2).

Another biological process that was significantly overrepresented in the parous control breast epithelia is that controlling gene transcription/gene transcription-regulation in which 21 genes were upregulated and 12 were downregulated (Tables I and II). Among these genes the Suppressor of Ty 5 homolog (SUPT5H), Homeo box D1 (HOXD1), p300/CBP associated factor (PCAF) and Inhibitor of DNA binding 4 (ID4) were highly upregulated in the control parous breast epithelia (Table I).

Genomic signature of the breast interlobular stroma. There were only 56 differentially expressed genes in the interlobular stroma from parous control women (Table III). The selection criteria was based on a B-value $>0$ and a $p<0.05$. We found 30 genes that were differentially overexpressed and 26
Table II. Downregulated genes in the parous control breast epithelia.

$\begin{array}{lr}\text { Apoptosis- GO:0006915 } & \\ \text { PDCD5 } & -2.1500 \\ \text { MDM4 } & -1.2500 \\ \text { Antiapoptosis- GO:0006916 } & -1.2600 \\ \text { BIRC6 } & -1.2700 \\ \text { BAG4 } & \\ \text { Cell adhesion- GO:0007154; GO:0007155; GO:0016337 } & -2.1000 \\ \text { DSCAM } & -1.8000 \\ \text { DLG5 } & -1.7800 \\ \text { COL16A1 } & -2.9100 \\ \text { LAMC1 }\end{array}$

Cell cycle and growth- GO:0007049; GO:0007050; GO:0000082; GO:0000074; GO:0000076; GO:0000086

$\begin{array}{lr}\text { KATNA1 } & -2.6600 \\ \text { TACC1 } & -2.1700 \\ \text { SESN3 } & -1.9000 \\ \text { GSPT1 } & -3.5000 \\ \text { PPP2R1B } & -2.3500 \\ \text { LATS1 } & -1.9800 \\ \text { TMCO7 } & -1.6000\end{array}$

Cell signaling- GO:0007267

Signal transduction- GO:0007165; GO:0007264; GO:0000160; GO:0000079; GO:0000186; GO:0005794

$\begin{array}{ll}\text { NPY1R } & -1.460 \\ \text { RAPGEF6 } & -1.4000 \\ \text { RAB27A } & -1.8454 \\ \text { CCDC132 } & -3.8868 \\ \text { BCCIP } & -1.3792 \\ \text { SCYE1 } & -3.8681 \\ \text { NPSR1 } & -1.4600 \\ \text { ANKDD1A } & -2.3100 \\ \text { EDNRA } & -1.4500 \\ \text { GIPC1 } & -1.5500 \\ \text { DDEF2 } & -1.3000\end{array}$

Cell transport - GO:0006886; GO:0006811; GO:0006817; GO:0006810; GO:0005794; GO:0006118; GO:001503; GO:0007242

$\begin{array}{ll}\text { STON2 } & -1.7224 \\ \text { GABRB3 } & -2.3657 \\ \text { FCN1 } & -1.6600 \\ \text { G3BP } & -3.8192 \\ \text { FREQ } & -1.4241 \\ \text { ACOX1 } & -1.5300 \\ \text { CYB5R4 } & -1.7000 \\ \text { RAP1B } & -1.8100 \\ \text { DBNDD2 } & -1.3100 \\ \text { SLC20A2 } & -1.7100 \\ \text { KLHL2 } & -2.0500 \\ \text { SNX11 } & -1.4600\end{array}$

Development-morphogenesis- GO:0009653; GO:0007275; GO:0007517; GO:0000188; GO:0007399; GO:0030326; GO:0007275; GO:0009790

TWIST1 
Table II. Continued.

\begin{tabular}{|c|c|}
\hline PIAS2 & -1.5000 \\
\hline MAP1B & -2.6405 \\
\hline DUSP22 & -1.6400 \\
\hline TPM3 & -2.2300 \\
\hline CRIM1 & -1.3500 \\
\hline SHFM1 & -1.7200 \\
\hline HLF & -1.5000 \\
\hline BRUNOL4 & -1.3400 \\
\hline \multicolumn{2}{|c|}{ DNA replication- GO:0006260; GO:0000067 } \\
\hline UBE1 & -1.250 \\
\hline SMC2 & -1.6100 \\
\hline \multicolumn{2}{|c|}{ Metabolism- GO:0005975; GO:0046677; GO:0008152 } \\
\hline AMY1A & -1.2500 \\
\hline ACSL3 & -1.2500 \\
\hline \multicolumn{2}{|c|}{ Miscellaneous processes- GO:0007596; GO:0046677; GO:0008152 } \\
\hline ANXA5 & -1.3938 \\
\hline LACTB & -1.8100 \\
\hline \multirow{2}{*}{\multicolumn{2}{|c|}{$\begin{array}{l}\text { Protein biosynthesis and metabolism- GO:0006470; GO:0006412; } \\
\text { GO:0006487; GO:0006461; GO:0006468; GO:0006457; GO:0018347 }\end{array}$}} \\
\hline & \\
\hline STYXL1 & -2.6900 \\
\hline MRPS16 & -1.6700 \\
\hline EIF2B1 & -1.4500 \\
\hline WARS2 & -1.7200 \\
\hline LIPC & -2.1300 \\
\hline PARD3 & -1.5500 \\
\hline TRPC4AP & -1.5700 \\
\hline CAPZA1 & -1.7800 \\
\hline COL4A3BP & -1.6700 \\
\hline MRPS11 & -1.6700 \\
\hline EIF1AY & -2.1500 \\
\hline FNTA & -1.7200 \\
\hline GRPEL2 & -1.6100 \\
\hline
\end{tabular}

Protein degradation and ubiquitination- GO:0016567; GO:0006508; GO:0006511; GO:0006512

$\begin{array}{ll}\text { SAE1 } & -1.5842 \\ \text { AFG3L2 } & -2.1800 \\ \text { TRIAD3 } & -2.0472 \\ \text { USP30 } & -1.7700 \\ \text { ARIH1 } & -2.1400 \\ \text { UBE2E1 } & -2.0700 \\ \text { RNA processing- GO:0000398; GO:0007046 } & \\ \text { SFRS10 } & -2.2000 \\ \text { BMS1L } & -1.6900 \\ \text { BXDC2 } & -2.1000\end{array}$

Transcription- GO:0000122; GO:0006355; GO:0006350; GO:0006306 HDAC8

ZNF425

$-1.6200$

PKNOX2

$-1.6400$

MBD3

$-3.1705$

GTF3C4

RNF12

DPF2

$-1.8700$

$-1.2500$

$-1.690$

SOX3
Table II. Continued.

\begin{tabular}{ll}
\hline POU6F1 & -1.3700 \\
MLLT6 & -1.4100 \\
RORA & -1.4000 \\
GATAD2A & -1.9700 \\
Biological process unknown- GO:0000004 & \\
PHACTR1 & -1.9118 \\
MGC4562 & -1.8500 \\
HNRPM & -1.7300 \\
RB1CC1 & -1.300 \\
DOCK5 & -1.3700 \\
DDX46 & -2.1900 \\
DOK5 & -1.2500 \\
PGRMC2 & -1.2500 \\
BCL7A & -1.3000 \\
ZNF320 & -1.5000 \\
NRSN2 & -4.8500 \\
TMEM32 & -4.4300 \\
ZRANB1 & -1.8500 \\
VKORC1L1 & -1.6600 \\
FAM57A & -3.8600 \\
MCPH1 & -3.9400 \\
\hline
\end{tabular}

genes that were downregulated in the parous control group (Table III). There was no biological process overrepresented and most of the Gene Ontology revealed unknown biological function.

\section{Discussion}

In the present study we demonstrate that early first full-term pregnancy imprints a specific genomic signature in the breast epithelia of postmenopausal women that is significantly different from that of women that also have had an early full-term pregnancy but have developed cancer. The genomic signature is made up of 126 upregulated and 103 downregulated genes. The gene ontology categories that were overrepresented in the breast epithelia of the parous control breast are related to apoptosis, DNA repair, response to exogenous agents, and gene transcription/gene transcriptionregulation.

There are 10 genes that control apoptosis that were differentially expressed in the breast epithelia of the parous women. Among them six were upregulated such as the BCL2-associated $X$ protein $(B A X)$ that belongs to the BCL2 protein family. $B A X$ is a pro-apoptotic gene whose transcription is stimulated by active p53, including the pro-apoptotic gene p21, a cell cycle regulator (21-23). This protein forms a heterodimer with BCL2, and functions as an apoptotic activator (24). The expression of this gene is regulated by the tumor suppressor P53 and has been shown to be involved in P53-mediated apoptosis $(21,25)$. Programmed cell death 5 (PDCD5) and Transformed $3 T 3$ cell double minute $4(\mathrm{Mdm} 4)$ were downregulated in the parous breast epithelia. The $M d m 4$ gene is amplified and overexpressed in a variety of human cancers and encodes structurally related oncoproteins that bind to the 
Table III. Gene expression profile of the stroma of the human breast.

\begin{tabular}{|c|c|c|c|c|c|c|c|c|}
\hline Gene name & Gene ID & Gene symbol & GO number & Biological function & GO number & Molecular function & Adj. p & Folds \\
\hline \multicolumn{9}{|l|}{ Genes Up-modulated } \\
\hline Apoptosis-inducing factor & H18472 & AMID & GO:0008637 & $\begin{array}{l}\text { Apoptotic, } \\
\text { mitochondrial }\end{array}$ & GO:0015036 & $\begin{array}{c}\text { Disulfide } \\
\text { oxidoreductase }\end{array}$ & 0.0354 & 1.5284 \\
\hline $\begin{array}{l}\text { Chromosome } 21 \text { open reading } \\
\text { frame } 45\end{array}$ & W72814 & C21orf45 & GO:0000004 & Unknown & GO:000554 & Unknown & 0.0001 & 1.2000 \\
\hline Hyaluronoglucosaminidase 1 & R44982 & HYAL1 & GO:0005975 & Metabolism & GO:000554 & Unknown & 0.0001 & 1.2871 \\
\hline Calcium channel & AA437099 & CACNA1D & GO:0006812 & Cation transport & GO:0005509 & Calcium ion binding & 0.0478 & 1.5600 \\
\hline $\begin{array}{l}\text { Hypothetical protein } \\
\text { LOC283874 }\end{array}$ & R01257 & LOC283874 & GO:0006313 & DNA transposition & GO:000554 & Unknown & 0.0001 & 1.2600 \\
\hline $\begin{array}{l}\text { Alcohol dehydrogenase } 6 \\
\text { (class V) }\end{array}$ & H68509 & ADH6 & GO:0006069 & Ethanol oxidation & GO:000554 & Unknown & 0.0001 & 1.2400 \\
\hline KIAA0319-like & AA150417 & KIAA0319L & GO:0007156 & Homophilic cell adhesion & GO:000554 & Unknown & 0.0219 & 3.8005 \\
\hline $\begin{array}{l}\text { SH3 and multiple ankyrin } \\
\text { repeat domains } 2\end{array}$ & R05837 & SHANK2 & GO:0007242 & Intracellular signaling & GO:000554 & Unknown & 0.0001 & 1.3255 \\
\hline $\begin{array}{l}\text { Translocase of outer } \\
\text { mitochondrial membrane } 40 \\
\text { homolog (yeast) }\end{array}$ & AA443094 & TOMM40 & GO:0006629 & Lipid metabolism & GO:000554 & Unknown & 0.0354 & 1.5786 \\
\hline $\begin{array}{l}\text { Retinoic acid receptor } \\
\text { responder (tazarotene } \\
\text { induced) } 1\end{array}$ & N94424 & RARRES1 & GO:0008285 & Cell proliferation & GO:000554 & Unknown & 0.0001 & 1.2400 \\
\hline Protein tyrosine kinase 2 & AA447612 & PTK2 & GO:0006468 & Protein phosphorylation & GO:0005524 & ATP binding & 0.0219 & 3.6419 \\
\hline $\begin{array}{l}\text { Regulator of G-protein } \\
\text { signalling } 12\end{array}$ & W67134 & RGS12 & GO:0015031 & Protein transport & GO:0005096 & GTPase activator & 0.0483 & 1.8100 \\
\hline Transcription factor Dp-1 & W46439 & TFDP1 & GO:0006357 & Transcription & GO:0003700 & Transcription factor & 0.0347 & 1.8023 \\
\hline $\begin{array}{l}\text { RNA pseudouridylate } \\
\text { synthase domain }\end{array}$ & H18934 & RPUSD1 & GO:0006396 & RNA processing & GO:0003723 & RNA binding & 0.0260 & 2.4640 \\
\hline $\begin{array}{l}\text { Colony stimulating factor } 2 \\
\text { receptor, } B, \text { low-affinity } \\
\text { (granulocyte-macrophage) }\end{array}$ & AA279147 & CSF2RB & GO:0007165 & Signal transduction & GO:000554 & Unknown & 0.0003 & 1.8900 \\
\hline Distal-less homeobox 5 & N74882 & DLX5 & GO:0001501 & Skeletal development & GO:000554 & Unknown & 0.0000 & 1.9794 \\
\hline $\begin{array}{l}\text { Adenosylmethionine } \\
\text { decarboxylase } 1\end{array}$ & AA504772 & AMD1 & GO:0006597 & Spermine biosynthesis & GO:000554 & Unknown & 0.0002 & 1.3800 \\
\hline $\begin{array}{l}\text { General transcription } \\
\text { factor IIIC }\end{array}$ & AA429809 & GTF3C4 & GO:0006350 & Transcription & GO:0003677 & DNA binding & 0.0260 & 3.1421 \\
\hline $\begin{array}{l}\text { Pleiomorphic adenoma } \\
\text { gene-like } 1\end{array}$ & AA463204 & PLAGL1 & GO:0006350 & Transcription & GO:000554 & Unknown & 0.0001 & 1.5600 \\
\hline Tripartite motif-containing 24 & R38345 & TRIM24 & GO:0006350 & Transcription & GO:000554 & Unknown & 0.0002 & 1.5500 \\
\hline $\begin{array}{l}\text { Transcription elongation } \\
\text { factor A (SII), } 2\end{array}$ & AA412500 & TCEA2 & GO:0006350 & Transcription & GO:000554 & Unknown & 0.0003 & 0.0981 \\
\hline ElaC homolog 1 (E. coli) & AA456439 & ELAC1 & GO:0006350 & Transcription & GO:000554 & Unknown & 0.0002 & 1.5600 \\
\hline $\begin{array}{l}\text { Transcobalamin II; } \\
\text { macrocytic anemia }\end{array}$ & AA490459 & TCN2 & GO:0006810 & Transport & GO:000554 & Unknown & 0.0002 & 1.4400 \\
\hline Similar to CG4502-PA & H17038 & FLJ25076 & GO:0006512 & Ubiquitin cycle & GO:000554 & Unknown & 0.0478 & 1.9000 \\
\hline $\begin{array}{l}\text { BRCA1 associated protein-1 } \\
\text { (ubiquitin carboxy-terminal } \\
\text { hydrolase) }\end{array}$ & H09066 & BAP1 & GO:0006511 & Ubiquitin-dependent & GO:000554 & Unknown & 0.0002 & 1.6400 \\
\hline $\begin{array}{l}\text { Transmembrane and } \\
\text { tetratricopeptide repeat }\end{array}$ & AA447480 & TMTC1 & GO:0000004 & Unknown & GO:0005488 & Binding & 0.0354 & 1.5128 \\
\hline $\begin{array}{l}\text { Hypothetical protein } \\
\text { LOC5 } 4103\end{array}$ & $\mathrm{~T} 82259$ & LOC54103 & GO:0000004 & Unknown & GO:000554 & Unknown & 0.0003 & 1.2400 \\
\hline C8orfK32 protein & H16974 & C8ORFK32 & GO:0000004 & Unknown & GO:000554 & Unknown & 0.0260 & 2.3494 \\
\hline Transcribed locus & N62346 & & GO:0000004 & Unknown & GO:000554 & Unknown & 0.0354 & 1.6003 \\
\hline $\begin{array}{l}\text { Transcription elongation } \\
\text { regulator 1-like }\end{array}$ & AA009615 & TCERG1L & GO:0000004 & Unknown & GO:000554 & Unknown & 0.0406 & 1.2500 \\
\hline Transcribed locus & AA010383 & & GO:0000004 & Unknown & GO:000554 & Unknown & 0.0260 & 2.5121 \\
\hline
\end{tabular}


Table III. Continued.

\begin{tabular}{|c|c|c|c|c|c|c|c|c|}
\hline Gene name & Gene ID & Gene symbol & GO number & Biological function & GO number & Molecular function & Adj. $\mathrm{p}$ & Folds \\
\hline $\begin{array}{l}\text { Genes down-modulated } \\
\text { Maternally expressed } \\
\text { (in Callipyge) } 8\end{array}$ & N52482 & MEG8 & GO:0000004 & Unknown & GO:000554 & Unknown & 0.0219 & -4.4853 \\
\hline KIAA1128 & AA432090 & KIAA1128 & GO:0000004 & Unknown & GO:000554 & Unknown & 0.0483 & -1.8000 \\
\hline $\begin{array}{l}\text { Hypothetical protein } \\
\text { DKFZp761B107 }\end{array}$ & R52679 & DKFZp761B107 & GO:0000004 & Unknown & GO:000554 & Unknown & 0.0335 & -1.9169 \\
\hline Clone 23688 mRNA sequence & N90403 & & GO:0000004 & Unknown & GO:000554 & Unknown & 0.0354 & -1.5971 \\
\hline Transcribed locus & AA431738 & & GO:0000004 & Unknown & GO:000554 & Unknown & 0.0481 & -1.8700 \\
\hline CDNA clone IMAGE:30404625 & H10156 & & GO:0000004 & Unknown & GO:000554 & Unknown & 0.0406 & -1.4100 \\
\hline Coiled-coil domain containing 46 & AA406069 & $\mathrm{CCDC} 46$ & GO:0000004 & Unknown & GO:000554 & Unknown & 0.0335 & -1.9632 \\
\hline Transcribed locus & AA426065 & & GO:0000004 & Unknown & GO:000554 & Unknown & 0.0260 & -2.6304 \\
\hline Tudor domain containing 10 & AA401393 & TDRD10 & GO:0000004 & Unknown & GO:000554 & Unknown & 0.0499 & -1.7300 \\
\hline Transcribed locus & R56233 & & GO:0000004 & Unknown & GO:000554 & Unknown & 0.0406 & -1.1970 \\
\hline Transcribed locus & N48294 & & GO:0000004 & Unknown & GO:000554 & Unknown & 0.0478 & -1.9300 \\
\hline Hypothetical protein P117 & AA005401 & P117 & GO:0000004 & Unknown & GO:000554 & Unknown & 0.0347 & -1.7668 \\
\hline $\begin{array}{l}\text { Seizure related } 6 \text { homolog } \\
\text { (mouse)-like }\end{array}$ & H29013 & SEZ6L & GO:0000004 & Unknown & GO:000554 & Unknown & 0.0335 & -1.9674 \\
\hline CDNA clone IMAGE:4800096 & AA428239 & & GO:0000004 & Unknown & GO:000554 & Unknown & 0.0260 & -2.7245 \\
\hline Transcribed locus & W32296 & & GO:0000004 & Unknown & GO:000554 & Unknown & 0.0381 & -1.4060 \\
\hline Transcribed locus & AA135722 & & GO:0000004 & Unknown & GO:000554 & Unknown & 0.0406 & -1.1710 \\
\hline $\begin{array}{l}\text { Thrombospondin, type I, } \\
\text { domain containing } 1 \text { pseudogene }\end{array}$ & AA115259 & THSD1P & GO:0000004 & Unknown & GO:000554 & Unknown & 0.0393 & -1.3400 \\
\hline $\begin{array}{l}\text { CDNA FLJ30588 fis, clone } \\
\text { BRAWH2008128 }\end{array}$ & T99852 & & GO:0000004 & Unknown & GO:000554 & Unknown & 0.0260 & -2.4091 \\
\hline WD repeat domain 68 & AA034041 & WDR68 & GO:0000004 & Unknown & GO:000554 & Unknown & 0.0260 & -2.4248 \\
\hline $\begin{array}{l}\text { Component of oligomeric golgi } \\
\text { complex } 6\end{array}$ & W67514 & COG6 & GO:0000004 & Unknown & GO:000554 & Unknown & 0.0002 & -1.3600 \\
\hline $\begin{array}{l}\text { 5'-nucleotidase domain } \\
\text { containing } 2\end{array}$ & $\mathrm{R} 42815$ & NT5DC2 & GO:0000004 & Unknown & GO:000554 & Unknown & 0.0002 & -1.4000 \\
\hline $\begin{array}{l}\mathrm{N} \text {-acylsphingosine amidohydrolase } \\
\text { (acid ceramidase)-like }\end{array}$ & W47576 & ASAHL & GO:0000004 & Unknown & GO:000554 & Unknown & 0.0001 & -1.4300 \\
\hline Small proline-rich protein $2 \mathrm{C}$ & AA399674 & SPRR2C & GO:0000004 & Unknown & GO:000554 & Unknown & 0.0001 & -1.5148 \\
\hline $\begin{array}{l}\text { Chromosome } 16 \text { open reading } \\
\text { frame } 61\end{array}$ & AA181314 & C16orf61 & GO:0000004 & Unknown & GO:000554 & Unknown & 0.0495 & -7.0000 \\
\hline
\end{tabular}

Adj. p, adjusted p-value.

p53 tumor suppressor protein and inhibit p53 activity (26-29). Mice with deleted Mdm4 die during embryogenesis, and the developmental lethality in this model can be rescued by concomitant deletion of p53 (30). The downregulation of the MDM4 in the breast of parous epithelia may act as a protective mechanism and be part of the program cell death pathway active in these cells. Altogether this cluster of genes seems to maintain the active programmed cell death pathway in the parous breast epithelia when compared with the parous breast of women with cancer. Supporting evidence for this statement comes from data in the experimental model (31-34) and in the normal breast tissue from reduction mammoplasty specimens of parous women (7-9), in which genes involved in the pathway of apoptosis were significantly upregulated.
We have identified in the present study that upregulation of DNA repair controlling genes is part of the signature induced by pregnancy. This is supported by data generated in the experimental system in which the parous mammary epithelial cells have a higher ability to remove the DNA adducts of 7-12 dimethylbenz (a) anthracene $(35,36)$. The greater ability of the parous mammary epithelial cells to remove the DNA adducts has been the first indication that an improved DNA repair was involved in the protective effect induced by pregnancy. DNA repair is central to the integrity of the human genome and reduced DNA repair capacity has been linked to genetic susceptibility to cancer (37-40). A reduced DNA repair is associated with risk of breast cancer in women (41). The epithelial cells of the breast from parous 
control women present DNA repair related genes that are upregulated significantly when compared with the same gene expression in the epithelial cells of the parous women with cancer. RAD51-like 3 was upregulated in the epithelial cells of the parous breast and it is known to be involved in the homologous recombination and repair of DNA (42-44). Other genes related to the DNA repair process are Ankyrin repeat domain 17 (ANKRD17) and Translin (TSN), which encodes a DNA-binding protein that specifically recognizes conserved target sequences at the breakpoint junction of chromosomal translocations (45). These data indicate that the activation of genes involved in the DNA repair process is part of the signature induced in the mammary gland by pregnancy, confirming previous findings that in vivo the ability of the cells to repair carcinogen-induced damage by unscheduled DNA synthesis and adduct removal is more efficient in the post-pregnancy mammary gland $(35,36)$.

Another cluster of genes that are upregulated in the parous control group are those related to immunosurveillance and detoxification of xenobiotic substances. The concept that an immunological process was involved during pregnancy which is responsible for its protective effect in mammary carcinogenesis has been reported $(46,47)$. In breast epithelial cells of parous postmenopausal women we found that the Toll-like receptor gene is upregulated. This gene belongs to the innate immune system recognizing microbial pathogens through Toll-like receptors (TLRs), which identify pathogenassociated molecular patterns $(48,49)$. We have also found that the regulatory factor $X$-associated protein that is part of the major histocompatibility (MHC) class II molecules (50) is upregulated (8). It is a transmembrane protein that has a central role in the development and control of the immune system. These data allow us to postulate that the increased immune-surveillance mechanism has been imprinted during the differentiation cycle induced by pregnancy and could be one of the protective factors induced by the cells against neoplastic initiation or progression.

In addition to this increase in the immune-surveillance mechanism in the breast of parous epithelia there are genes significantly upregulated and involved in the metabolism of xenobiotic substances and oxidative stress. Among them are the Epoxide hydrolase (EPHX1) that plays an important role in both the activation and detoxification of exogenous chemicals such as polycyclic aromatic hydrocarbons and the Thioredoxin reductase 1 (TXNRD1) that encodes a member of a family of pyridine nucleotide oxidoreductases. TXNRD1 protein reduces thioredoxins as well as other substrates, and plays a role in selenium metabolism and protection against oxidative stress $(51,52)$. Thioredoxin reductase 1 is one of the major antioxidant and redox regulators in mammals that supports p53 function and other tumor suppressor activities $(53,54)$. Glutathione $S$ transferase $\theta 1$ (GSTT1) is a member of a superfamily of proteins that catalyzes the conjugation of reduced glutathione to a variety of electrophilic and hydrophobic compounds and is upregulated in the parous breast epithelia. The other gene that is also overexpressed is the $\mathrm{N}$-acetyltransferase 2 [arylamine $N$-acetyltransferase (NAT2)] involved in the metabolism of different xenobiotics, including potential carcinogens. The upregulation of these genes is interpreted as an activated system of defense that makes the parous breast cells less vulnerable to genotoxic substances. This contention is supported by data indicating that primary breast epithelial cells from parous women treated in vitro with chemical carcinogens do not express phenotypes of cell transformation whereas those from nulliparous women do $(55,56)$.

There are 21 genes encoding proteins controlling gene transcription/gene transcription-regulation that are significantly upregulated in the parous breast epithelia and 12 that are downregulated. This indicates that during pregnancy transcription modifications are important components of the genomic signature induced by this physiological process. Another group of genes associated with their function as coactivator and in chromatin remodeling seems to play an important role in the signature induced by pregnancy in the breast epithelial cells. One of them is the $p 300 / C B P$-associated factor $(P C A F)$ that is significantly upregulated in the epithelial cells of the parous breast tissue (7-9). PCAF is a coactivator of the tumor suppressor, p53. PCAF participates in p53's transactivation of target genes through acetylation of both bound p53 and histones within p53 target promoters (57). The role of $p 300 / C B P$-associated factor in the differentiated breast epithelial cells of parous women could be similar to the effect of trans-retinoic acid (ATRA) treatment on metastatic breast cancer cells that, by increasing the protein levels of the histone acetyl transferases p300 and CBP, suppresses the level of histone deacetylase and increases the level of acetylated histone H4. ATRA also has been shown to decrease Bcl-2 and VEGF and increase $B A X$ (58). $B A X$ is upregulated in the parous breast epithelial cells. $P C A F$ has been considered part of the genomic signature of the Stem cell 2 (7-9).

ID4 (Inhibitor of DNA binding 4) is a member of the Id family of proteins (Id1-Id4), which function as dominantnegative regulators of basic helix-loop-helix transcription factors and are involved in numerous cell processes, including cell proliferation, and differentiation (59). Id4 is constitutively expressed in the normal human mammary epithelium but is suppressed in breast carcinomas and preneoplastic lesions supporting a possible role of Id4 as a tumor suppressor factor in the human breast $(59,60)$. Primary breast cancers have low or no expression of ID4 protein (61) and ID4 has also been considered a putative tumor-suppressor gene that is methylated in most mouse and human leukemias (62-65).

Altogether our data indicate that early first full-term pregnancy induces in the breast epithelia a specific genomic profile that can be identified in the postmenopausal breast and that makes these epithelial cells different to parous breast tissue from women with breast cancer. This genomic signature allows us to evaluate the degree of mammary gland differentiation induced by pregnancy and it could be the signature postulated for the Stem cell $2(7-9,66)$. This signature could help to predict in which woman parity is protective, and it can be used as a biomarker for evaluating preventive agents.

\section{Acknowledgments}

This study was supported by Grant RO1-CA093599 from the National Cancer Institute, USA. The authors thank Dr Joanna Dorgan for supervising the breast tissue and data collection and Debra Riordan MS, Ryan Hopson MS and Irene Shandruk MS at FCCC, Gladwyn Downes from Christiana Health Care 
and Barbara Carney from Somerset Medical Center for collecting the clinical data of all the patients entered in this study.

\section{References}

1. Greenlee RT, Murray T, Boldin S and Wingo P: Cancer statistics, 2000. Ca Cancer J Clin 50: 7-23, 2000.

2. MacMahon B, Cole P, Lin TM, et al: Age at first birth and breast cancer risk. Bull Natl Hlth Org 34: 209-221, 1970.

3. Vessey MD, McPherson K, Roberts MM, Neil A and Jones L: Fertility and the risk of breast cancer. Br J Cancer 52: 625-628, 1985.

4. Trapido EJ: Age at first birth, parity and breast cancer risk. Cancer 51: 946-948, 1983.

5. Russo J, Tay LK and Russo IH: Differentiation of the mammary gland and susceptibility to carcinogenesis: A Review. Breast Cancer Res Treat 2: 5-73, 1982.

6. De Waard F and Trichopoulos D: A unifying concept of the etiology of breast cancer. Int J Cancer 41: 666-669, 1988.

7. Russo J, Balogh GA, Chen J, Fernandez SV, et al: The concept of stem cell in the mammary gland and its implication in morphogenesis, cancer and prevention. Front Biosci 11: 151-172, 2006.

8. Balogh GA, Heulings R, Mailo DA, Russo J, et al: Genomic signature induced by pregnancy in the human breast. Int $\mathrm{J}$ Oncology 28: 399-410, 2006.

9. Russo J, Balogh GA, Heulings R, et al: Molecular basis of pregnancy induced breast cancer protection. Eur J Cancer Prev 15: 306-342, 2006

10. Russo J and Russo IH: Development of the human mammary gland. In: The Mammary Gland. Neville MC and Daniel C (eds). Plenum Publishing Corporation, New York, NY, pp67-93, 1987.

11. Russo J, Rivera R and Russo J: Influence of age and parity on the development of the human breast. Breast Cancer Res Treat 23: 211-218, 1992.

12. Balogh GA, Heulings R, Russo IH, Mailo D, Li Y-S and Russo J: Methodological approach to study the genomic profile of the human breast. Int J Oncol 31: 253-260, 2007.

13. Gentleman R, Bates D, Bolstad B, et al: Bioconductor: open software development for computational biology and bioinformatics. Genome Biol 5: R80, 2004.

14. Wettenhall JM, Smyth GK and Limma G: A graphical user interface for linear modeling of microarray data. Bioinformatics 20: 3705-3706, 2004.

15. Irizarry RA, Hobbs B, Collin F, et al: Exploration, normalization and summaries of high density oligonucleotide array probe level data. Biostatistics 4: 249-264, 2003.

16. Smyth GK and Speed T: Normalization of cDNA microarray data. Methods 31: 265-273, 2003.

17. Smyth GK: Limma linear models for microarray data. In: Bioinformatics and Computational Biology Solutions using $\mathrm{R}$ and Bioconductor. Gentleman R, Carey V, Dudoit S, Irizarry R, and Huber W (eds). Springer, New York, pp397-420, 2005.

18. Smyth GK, Michaud J and Scott H: The use of within-array replicate spots for assessing differential expression in microarray experiments. Bioinformatics 21: 2067-2075, 2005.

19. Ritchie ME, Diyagama D, Neilson J, et al: Empirical array quality weights for microarray data. BMC Bioinformatics 7 : 261-267, 2006.

20. Smyth GK: Linear models and empirical Bayes methods for assessing differential expression in microarray experiments. Stat Appl Genet Mol Biol 3: Article 3, 2004.

21. Cheng J, Cui R, Chen $\mathrm{CH}$ and Du J: Oxidized low-density lipoprotein stimulates p53-dependent activation of proapoptotic Bax leading to apoptosis of differentiated endothelial progenitor cells. Endocrinology 148: 2085-2094, 2007.

22. Shankar S and Srivastava RK: Bax and Bak genes are essential for maximum apoptotic response by curcumin, a polyphenolic compound and cancer chemopreventive agent derived from turmeric, Curcuma longa. Carcinogenesis 28: 1277-1286, 2007.

23. Lysiak JJ, Zheng S, Woodson R and Turner TT: Caspase-9dependent pathway to murine germ cell apoptosis: mediation by oxidative stress, BAX, and caspase 2 . Cell Tissue Res 328: 411-419, 2007.

24. Eissing T, Waldherr S, Allgower F, Scheurich P and Bullinger E: Response to bistability in apoptosis: roles of bax, bcl-2, and mitochondrial permeability transition pores. Biophys J 92: 3332-3334, 2007.
25. Geng Y, Akhtar RS, Shacka JJ, Klocke BJ, Zhang J, Chen X and Roth KA: p53 transcription-dependent and -independent regulation of cerebellar neural precursor cell apoptosis. Neuropathol Exp Neurol 66: 66-74, 2007.

26. Boesten LS, Zadelaar SM, De Clercq S, et al: Mdm2, but not $\mathrm{Mdm} 4$, protects terminally differentiated smooth muscle cells from p53-mediated caspase-3-independent cell death. Cell Death Diff 13: 2089-2098, 2006.

27. Toledo F, Krummel KA, Lee CJ, Liu CW, Rodewald LW, Tang $M$ and Wahl GM: A mouse p53 mutant lacking the proline-rich domain rescues $\mathrm{Mdm} 4$ deficiency and provides insight into the Mdm2-Mdm4-p53 regulatory network. Cancer Cell 9: 273-285, 2006.

28. Francoz S, Froment P, Bogaerts S, et al: Mdm4 and Mdm2 cooperate to inhibit p53 activity in proliferating and quiescent cells in vivo. Proc Natl Acad Sci USA 103: 3232-3237, 2006.

29. Xiong S, Van Pelt CS, Elizondo-Fraire AC, Liu G and Lozano G: Synergistic roles of $\mathrm{Mdm} 2$ and Mdm4 for p53 inhibition in central nervous system development. Proc Natl Acad Sci USA 103: 3226-3231, 2006.

30. Steinman HA, Hoover KM, Keeler ML, Sands AT and Jones SN: Rescue of Mdm4-deficient mice by Mdm2 reveals functional overlap of Mdm2 and Mdm4 in development. Oncogene 24: 7935-7940, 2005.

31. Russo J, Mailo D, Hu YF, Balogh GA, Sheriff F and Russo IH: Breast differentiation and its implication in cancer prevention. Clin Cancer Res 11: 931s-936s, 2005.

32. Srivastava P, Russo J and Russo IH: Chorionic gonadotropin inhibits rat mammary carcinogenesis through activation of programmed cell death. Carcinogenesis 18: 1799-1808, 1997.

33. Srivastava P, Russo J and Russo IH: Inhibition of rat mammary tumorigenesis by human chorionic gonadotropin is associated with increased expression of inhibin. Mol Carcinog 26: 1-10, 1999.

34. Russo J and Russo IH: Human chorionic gonadotropin in breast cancer prevention. In: Endocrine Oncology. Ethier SP (ed). Humana Press Inc, Totowa NJ, pp121-136, 2000.

35. Tay LK and Russo J: 7, 12-Dimethylbenz (a) anthracene (DMBA) induced DNA binding and repair synthesis in susceptible and non-susceptible mammary epithelial cells in culture. J Natl Cancer Inst 67: 155-161, 1981.

36. Tay LK and Russo J: Formation and removal of 7,12dimethylbenz(a)- anthracene-nucleic acid adducts in rat mammary epithelial cells with different susceptibility to carcinogenesis. Carcinogenesis 2: 1327-1333, 1981.

37. Bindra RS and Glazer PM: Co-repression of mismatch repair gene expression by hypoxia in cancer cells: Role of the Myc/Max network. Cancer Lett 252: 93-103, 2007.

38. Materna V, Surowiak P, Markwitz E, Spaczynski M, DragZalesinska M, Zabel M and Lage H: Expression of factors involved in regulation of DNA mismatch repair and apoptosis pathways in ovarian cancer patients. Oncol Rep 17: 505-516, 2007.

39. Tudek B, Swoboda M, Kowalczyk P and Olinski R: Modulation of oxidative DNA damage repair by the diet, inflammation and neoplastic transformation. J Physiol Pharmacol 57: 33-49, 2006.

40. Bonde P, Gao D, Chen L, et al: Selective decrease in the DNA base excision repair pathway in squamous cell cancer of the esophagus. J Thorac Cardiovasc Surg 133: 74-81, 2006.

41. Shi Q, Wang LE, Bondy ML, Brewster A, Singletary SE and Wei Q: Reduced DNA repair of benzo[a]pyrene diol epoxideinduced adducts and common XPD polymorphisms in breast cancer patients. Carcinogenesis 25: 1695-1700, 2004.

42. Wiese C, Hinz JM, Tebbs RS, et al: Disparate requirements for the Walker A and B ATPase motifs of human RAD51D in homologous recombination. Nucleic Acid Res 34: 2833-2843, 2006.

43. Gruver AM, Miller KA, Rajesh C, et al: The ATPase motif in RAD51D is required for resistance to DNA interstrand crosslinking agents and interaction with RAD51C. Mutagenesis 20: 433-440, 2005.

44. Kawabata M, Kawabata T and Nishibori M: Role of recA/RAD51 family proteins in mammals. Acta Med Okayama 59: 1-9, 2005.

45. Mellon SH, Bair SR, Depoix C, Vigne JL, Hecht NB and Brake P: Translin coactivates steroidogenic factor-1-stimulated transcription. Mol Endocrinol 21: 89-105, 2007.

46. Sinha DK, Pazik JE and Dao TL: Prevention of mammary carcinogenesis in rats by pregnancy: effect of full-term and interrupted pregnancy. Br J Cancer 57: 390-394, 1988. 
47. D'Cruz CM, Moody SE, Master SR, et al: Persistent parityinduced changes in growth factors, TGF-beta3, and differentiation in the rodent mammary gland. Mol Endocrinol 16: 2034-2051, 2002.

48. Sanghavi SK, Shankarappa R and Reinhart TA: Genetic analysis of Toll/Interleukin-1 Receptor (TIR) domain sequences from rhesus macaque Toll-like receptors (TLRs) 1-10 reveals high homology to human TLR/TIR sequences. Immunogenetics 56 : 667-674, 2004.

49. Yamamoto $\mathrm{M}$ and Akira S: TIR domain containing adaptors regulate TLR-mediated signaling pathways. Nippon Rinsho 62: 65-72, 2004.

50. Kim M, Li D, Cui Y, Mueller K, Chears WC and DeJong J: Regulatory factor interactions and somatic silencing of the germ cell-specific ALF gene. J Biol Chem 281: 34288-34298, 2006.

51. Yoon BI, Kim DY, Jang JJ and Han JH: Altered expression of thioredoxin reductase-1 in dysplastic bile ducts and cholangiocarcinoma in a hamster model. J Vet Sci 7: 211-216, 2006.

52. Yegorova S, Yegorov O and Lou MF: Thioredoxin induced antioxidant gene expressions in human lens epithelial cells. Exp Eye Res 83: 783-792, 2006.

53. Yoo MH, Xu XM, Carlson BA, Gladyshev VN and Hatfield DL: Thioredoxin reductase 1 deficiency reverses tumor phenotype and tumorigenicity of lung carcinoma cells. J Biol Chem 281: 13005-13008, 2006.

54. Rigobello MP, Vianello F, Folda A, Roman C, Scutari G and Bindoli A: Differential effect of calcium ions on the cytosolic and mitochondrial thioredoxin reductase. Biochem Biophys Res Commun 343: 873-888, 2006

55. Russo J, Reina D, Frederick J and Russo IH: Expression of phenotypical changes by human breast epithelial cells treated with carcinogens in vitro. Cancer Res 48: 2837-2857, 1988

56. Russo J, Calaf G and Russo IH: A critical approach to the malignant transformation of human breast epithelial cells. Crit Rev Oncog 4: 403-417, 1993.
57. Watts GS, Oshiro MM, Junk DJ, et al: The acetyltransferase p300/CBP-associated factor is a p53 target gene in breast tumor cells. Neoplasia 6: 187-194, 2004.

58. Hayashi K, Goodison S, Urquidi V, Tarin D, Lotan R and Tahara E: Differential effects of retinoic acid on the growth of isogenic metastatic and non-metastatic breast cancer cell lines and their association with distinct expression of retinoic acid receptor beta isoforms 2 and 4. Int J Oncol 22: 623-629, 2003.

59. de Candia P, Akram M, Benezra R and Brogi E: Id4 messenger RNA and estrogen receptor expression: inverse correlation in human normal breast epithelium and carcinoma. Hum Pathol 37: 1032-1041, 2006.

60. Roldan G, Delgado L and Muse IM: Tumoral expression of BRCA1, estrogen receptor alpha and ID4 protein in patients with sporadic breast cancer. Cancer Biol Ther 5: 505-510, 2006.

61. Umetani N, Mori T, Koyanagi K, Shinozaki M, Kim J, Giuliano AE and Hoon DS: Aberrant hypermethylation of ID4 gene promoter region increases risk of lymph node metastasis in T1 breast cancer. Oncogene 24: 4721-4727, 2005.

62. Yu L, Liu C, Vandeusen J, et al: Global assessment of promoter methylation in a mouse model of cancer identifies ID4 as a putative tumor-suppressor gene in human leukemia. Nat Genet 37: 265-274, 2005.

63. Umetani N, Takeuchi H, Fujimoto A, Shinozaki M, Bilchik AJ and Hoon DS: Epigenetic inactivation of ID4 in colorectal carcinomas correlates with poor differentiation and unfavorable prognosis. Clin Cancer Res 10: 7475-7483, 2004.

64. de Candia P, Benera R and Solit DB: A role for Id proteins in mammary gland physiology and tumorigenesis. Adv Cancer Res 92: 81-94, 2004

65. Chan AS, Tsui WY, Chen X, Chu KM, et al: Downregulation of ID4 by promoter hypermethylation in gastric adenocarcinoma. Oncogene 22: 6946-6953, 2003.

66. Russo J and Russo IH: Role of differentiation in the pathogenesis and prevention of breast cancer. Endocr Rel Cancer 4: 1-15, 1997. 